\title{
THE TIME-DEPENDENT STOKES PARADOX
}

\author{
BY
}

S. H. SMITH

University of Toronto, Toronto, Ontario, Canada

\begin{abstract}
When a uniform stream starts to flow impulsively at time $t=0$ past a two-dimensional body, the solution of the Stokes equation indicates that the velocity grows without bound as $t \rightarrow \infty$. It is seen that this is a natural extension of the Stokes paradox to unsteady flows. Two resolutions of this result are presented: firstly, through an analysis of the Oseen equation on the basis of singular perturbation theory and, secondly, through considering the two-dimensional body as the limit of a threedimensional body when the length increases without bound.
\end{abstract}

When Stokes [1] tried to describe the uniform streaming flow past a circular cylinder, he found it was impossible to find a solution of the governing two-dimensional equations for slow viscous flow which gave finite velocities at large distances-a conclusion now known as the "Stokes paradox." Although Oseen showed that the velocity is bounded when the convection terms are included for $0<R e \ll 1$, the full resolution of the paradox required the perspective of singular perturbation theory as developed by Kaplun and Lagerstrom [2], and Proudman and Pearson [3]. They showed that the Stokes equation is only valid in the inner domain where the radial distance $r=O(1)$, whereas the Oseen equation is appropriate in the outer domain where $\operatorname{Re} r=O(1)$; the solutions in the separate regions can then be matched across the boundary where $r \rightarrow \infty, \operatorname{Re} r \rightarrow 0$.

In his classical paper, Stokes writes (in paragraph 46)

We have evidently a right to conceive a sphere or infinite cylinder to exist at rest in an infinite mass of fluid also at rest, to suppose the sphere or cylinder to be then moved with a uniform velocity $V$, and to propose for determination the motion of the fluid at the end of the time $t$. But we have no right to assume that the motion approaches a permanent state as $t$ increases indefinitely. We may follow either of two courses. We may proceed to solve the general problem in which the sphere or cylinder is supposed to move from rest, and then examine what results we obtain by supposing $t$ to increase indefinitely, or else we may assume for trial that the motion is steady, and proceed to inquire whether we can satisfy all the conditions of the problem on

Received June 13, 1989.

(C)1991 Brown University 
this supposition. The former course would have the disadvantage of requiring a complicated analysis for the sake of obtaining a comparatively simple result, and it is even possible that the solution of the problem might baffle us altogether; but if we adopt the latter course, we must not forget that the equations with which we work are only provisional.

In the first part of this paper we follow the former course through considering the time-dependent behaviour after an impulsive start at time $t=0$. Rather than consider a finite body, with the attendant problems (fully realised by Stokes) of trying to satisfy boundary conditions, we take advantage of generalized functions (unavailable to him) and consider the flow past a point source of momentum-a stokeslet or oseenlet-and find that this model is able to display the basic phenomenon.

The velocities are written as $u(x, y, t)$ and $v(x, y, t)$ in the $x$-and $y$-directions, respectively; $p(x, y, t)$ is the pressure. The fluid with density $\rho$ and viscosity $\nu$ flows with velocity $U$ in the $x$-direction, when a point source of momentum is created at time $t=0$. The Oseen equations of motion for the slow viscous flow are

$$
\begin{aligned}
u_{x}+v_{y} & =0, \\
u_{t}+U u_{x} & =-\rho^{-1} p_{x}+\nu \nabla^{2} u+\nu U \delta(x) \delta(y) H(t), \\
v_{t}+U v_{x} & =-\rho^{-1} p_{y}+\nu \nabla^{2} v
\end{aligned}
$$

the coefficient of the generalized function is fixed to ensure the correct dimensions only. When a stream function $\psi(x, y, t)$ is introduced through $u=\psi_{y}, v=-\psi_{x}$, then the pressure can be eliminated for

$$
\omega_{t}+U \omega_{x}=\nu \nabla^{2} \omega+\nu U \delta(x) \delta^{\prime}(y) H(t), \quad \omega=\nabla^{2} \psi .
$$

This equation can be solved completely through defining the Fourier transforms in $x, y$, plus the Laplace transform in $t$ by

$$
\bar{\psi}(\alpha, \beta, s)=\int_{-\infty}^{\infty} e^{-i \beta y} d y \int_{-\infty}^{\infty} e^{-i \alpha x} d x \int_{0}^{\infty} e^{-s t} \psi(x, y, t) d t .
$$

However, we commence by first neglecting the convection term $U \omega_{x}$ in (1), thereby giving the unsteady Stokes equation, and when the transform (2) is taken it follows that the solution $\psi_{s}(x, y, t)$ is given by

$$
\bar{\psi}_{s}=-\frac{i \nu U \beta}{s\left(\alpha^{2}+\beta^{2}\right)\left\{s+\nu\left(\alpha^{2}+\beta^{2}\right)\right\}} .
$$

Here the integrals in the inverse transform can all be evaluated from standard tables (cf. Erdelyi et al [4]) for

$$
\psi_{s}(x, y, t)=\frac{\nu U t y}{2 \pi r^{2}}\left\{1-\exp \left(-\frac{r^{2}}{4 \nu t}\right)\right\}-\frac{U y}{8 \pi} E i\left(-\frac{r^{2}}{4 \nu t}\right),
$$


where $E i(-z)=-\int_{z}^{\infty} v^{-1} e^{-v} d v$ is the exponential integral for $z>0$. The corresponding expression for the vorticity $\omega_{s}$ is given by

$$
\omega_{s}(x, y, t)=-\frac{U y}{2 \pi r^{2}} \exp \left(-\frac{r^{2}}{4 \nu t}\right) .
$$

Now it is known that $E i(-z)=\ln z-z+O\left(z^{2}\right)$ as $z \rightarrow 0+$, and so it is seen that $\psi$ grows without bound as $\frac{1}{8} U \pi^{-1} y \ln (\nu t)$ for $r=O(1)$ as $t \rightarrow \infty$; that is, a uniform stream is present whose velocity becomes logarithmically large in time. This result follows from the fact that diffusion is the only process included in the Stokes equation, which acts through the variable $r^{2} / \nu t$. When the steady state equation

$$
0=\nabla^{2} \omega+U \delta(x) \delta^{\prime}(y), \quad \omega=\nabla^{2} \psi
$$

is solved, and the solution which follows is written as $\psi_{s \infty}$, then

$$
\psi_{s \infty}=-\frac{U y}{4 \pi}(\ln r+C)
$$

for some constant $C$, and the $y \ln r$ term is present as anticipated for the Stokes paradox. The $\ln t$ singularity as $t \rightarrow \infty$ for the unsteady flow is therefore an inevitable consequence of the Stokes paradox. Physically, it can be seen that the timedependent Stokes equation is attempting to describe a process whereby a finite force acts through a finite time $t$ to produce a motion with infinite energy; consequently, no steady flow will be reached when the reduced equation is considered.

Not that this is altogether an original conclusion, for to quote Stokes once more (from paragraph 47)

As the cylinder moves on, it carries more and more of the fluid with it, in consequence of friction... . The pressure of the cylinder on the fluid continually tends to increase the quantity of fluid which it carries with it, while the friction of the fluid at a distance from the cylinder continually tends to diminish it. In the case of a sphere, these two causes eventually counteract each other, and the motion becomes uniform. But in the case of a cylinder, the increase in the quantity of fluid carried continually gains on the decrease due to the friction of the surrounding fluid, and the quantity carried increases indefinitely as the cylinder moves on.

He clearly had a physical understanding of the role of the diffusion variable, long before Rayleigh had presented his calculations.

To try and resolve the situation, we now solve the complete Eq. (1), including the convection term. Again, Stokes suspected that the neglect of convection might be responsible for the difficulty, commenting

... it may not be safe in such an extreme case to neglect the terms depending on the square of the velocity, not that they become unusually large in themselves, but only unusually large compared with the terms retained, ... 
(in paragraph 48). To solve (1), we use the transform (2) and it is seen that the expression for the stream function $\psi_{o}(x, y, t)$ is given by

$$
\bar{\psi}_{o}=-\frac{i U \nu \beta}{s\left(\alpha^{2}+\beta^{2}\right)\left\{s+\nu\left(\alpha^{2}+\beta^{2}+i U \alpha\right)\right\}} .
$$

Only two of the integrals in the inverse transform can be evaluated, and we are left with

$$
\begin{aligned}
\psi_{o}= & \frac{\nu}{2 \pi}\left\{\arctan \left(\frac{x}{y}\right)-\arctan \left(\frac{x-U t}{y}\right)\right\} \\
+ & \frac{\nu}{4 \pi} \int_{0}^{\infty} \frac{\sin \alpha(x-U t)}{\alpha}\left[e^{\alpha y} \operatorname{erfc}\left\{\frac{y}{2(\nu t)^{1 / 2}}+\alpha(\nu t)^{1 / 2}\right\}\right. \\
& \left.+e^{-\alpha y} \operatorname{erfc}\left\{\frac{y}{2(\nu t)^{1 / 2}}-\alpha(\nu t)^{1 / 2}\right\}\right] \\
& -\frac{\nu e^{\kappa x}}{4 \pi} \int_{0}^{\infty} \frac{\alpha \sin \alpha x+\kappa \cos \alpha x}{\alpha^{2}+\kappa^{2}} \\
& \times\left[e^{\left(\alpha^{2}+\kappa^{2}\right)^{1 / 2} y} \operatorname{erfc}\left\{\frac{y}{2(\nu t)^{1 / 2}}+\left(\alpha^{2}+\kappa^{2}\right)^{1 / 2}(\nu t)^{1 / 2}\right\}\right. \\
& \left.\quad+e^{-\left(\alpha^{2}+\kappa^{2}\right)^{1 / 2} y} \operatorname{erfc}\left\{\frac{y}{2(\nu t)^{1 / 2}}-\left(\alpha^{2}+\kappa^{2}\right)^{1 / 2}(\nu t)^{1 / 2}\right\}\right] d \alpha,
\end{aligned}
$$

and

$$
\begin{aligned}
\omega_{o}=-\frac{U e^{\kappa x}}{4 \pi} \int_{0}^{\infty} \cos \alpha x\left[e^{\left(\alpha^{2}+\kappa^{2}\right)^{1 / 2} y} \operatorname{erf} c\left\{\frac{y}{2(\nu t)^{1 / 2}}+\left(\alpha^{2}+\kappa^{2}\right)^{1 / 2}(\nu t)^{1 / 2}\right\}\right. \\
\left.+e^{-\left(\alpha^{2}+\kappa^{2}\right)^{1 / 2} y} \operatorname{erfc}\left\{\frac{y}{2(\nu t)^{1 / 2}}-\left(\alpha^{2}+\kappa^{2}\right)^{1 / 2}(\nu t)^{1 / 2}\right\}\right] d \alpha
\end{aligned}
$$

where $\kappa=\frac{1}{2} U \nu^{-1}$. However, the integral for the time derivative of (8) can be evaluated for the simple expression

$$
\omega_{o t}=-\frac{U y}{8 \pi \nu t^{2}} \exp \left\{-\frac{(x-U t)^{2}+y^{2}}{4 \nu t}\right\} ;
$$

this represents a pulse being convected downstream with velocity $U$, while diffusion acts to spread the effect radially outwards. For $x \gg U t$ diffusion dominates and the role of convection is negligible; for $x \ll U t$ there is exponential decay as $\exp \left(-\frac{1}{4} U^{2} t / \nu\right)$ to the steady state. Also, the integration can be completed for large time with

$$
\left(\omega_{o}\right)_{t \rightarrow \infty}=-\frac{U^{2} y}{4 \pi \nu r} e^{\kappa x} K_{1}(\kappa r),
$$

to give the vorticity for the oseenlet, as is well known. 
To analyze the stream function, it is easiest to investigate the velocity $\psi_{o y}$ and, after some simplification of the derivative of (7), we find that

$$
\begin{aligned}
\psi_{o y}= & \frac{\nu}{2 \pi}\left[\frac{x-U t}{(x-U t)^{2}+y^{2}}\left\{1-\exp \left(-\frac{(x-U t)^{2}+y^{2}}{4 \nu t}\right)\right\}-\frac{x}{x^{2}+y^{2}}\right] \\
- & \frac{\nu}{2 \pi} e^{\kappa x} \int_{0}^{\infty} \frac{\alpha \sin \alpha x+\kappa \cos \alpha x}{\left(\alpha^{2}+\kappa^{2}\right)^{1 / 2}} \\
& \times\left[e^{\left(\alpha^{2}+\kappa^{2}\right)^{1 / 2} y} \operatorname{erfc}\left\{\frac{y}{2(\nu t)^{1 / 2}}+\left(\alpha^{2}+\kappa^{2}\right)^{1 / 2}(\nu t)^{1 / 2}\right\}\right. \\
& \left.-e^{-\left(\alpha^{2}+\kappa^{2}\right)^{1 / 2} y} \operatorname{erfc}\left\{\frac{y}{2(\nu t)^{1 / 2}}-\left(\alpha^{2}+\kappa^{2}\right)^{1 / 2}(\nu t)^{1 / 2}\right\}\right] d \alpha .
\end{aligned}
$$

To relate this integral more precisely with the Stokes solution, it is helpful to introduce the scaled diffusion variables

$$
\rho=\frac{r}{2(\nu t)^{1 / 2}}, \quad \eta=\frac{x}{2(\nu t)^{1 / 2}}, \quad \zeta=\frac{y}{2(\nu t)^{1 / 2}}, \quad \text { plus } \quad \tau=\frac{U}{2}\left(\frac{t}{\nu}\right)^{1 / 2} ;
$$

then

$$
\begin{aligned}
\psi_{s y}= & \frac{U}{8 \pi}\left[\frac{\cos 2 \theta}{\rho^{2}}\left(1-e^{-\rho^{2}}\right)-E i\left(-\rho^{2}\right)\right], \quad \tan \theta=\frac{y}{x}, \\
\psi_{o y}= & \frac{1}{4 \pi}\left(\frac{\nu}{t}\right)^{1 / 2}\left[\frac{\eta-\tau}{(\eta-\tau)^{2}+\zeta^{2}}\left\{1-e^{-(\eta-\tau)^{2}-\zeta^{2}}\right\}-\frac{\eta}{\rho^{2}}\right] \\
& -\frac{e^{2 \tau \eta}}{8 \pi}\left(\frac{\nu}{t}\right)^{1 / 2} \int_{0}^{\infty} \frac{\gamma \sin \gamma \eta+2 \tau \cos \gamma \eta}{\left(\gamma^{2}+4 \tau^{2}\right)^{1 / 2}} \\
& \times\left\{e^{\left(\gamma^{2}+4 \tau^{2}\right)^{1 / 2} \zeta} \operatorname{erfc}\left[\zeta+\frac{1}{2}\left(\gamma^{2}+4 \tau^{2}\right)^{1 / 2}\right]\right. \\
& \left.-e^{\left(\gamma^{2}+4 \tau^{2}\right)^{1 / 2} \zeta} \operatorname{erfc}\left[\zeta-\frac{1}{2}\left(\gamma^{2}+4 \tau^{2}\right)^{1 / 2}\right]\right\} d \gamma .
\end{aligned}
$$

It follows when

$$
\tau \gg 1, \quad \rho \ll 1 \quad \text { with } \tau \rho=O(1),
$$

that the dominant contribution towards $\psi_{o y}$ is

$$
\psi_{o y} \simeq-\frac{1}{4 \pi}\left(\frac{\nu}{t}\right)^{1 / 2} \frac{\eta}{\rho^{2}}+\frac{U}{4 \pi} e^{2 \tau \eta}\left\{\frac{\eta}{\rho} K_{1}(2 \tau \rho)+K_{0}(2 \tau \rho)\right\} .
$$

Now $2 \tau \rho=\frac{1}{2} U r / \nu$, and so (13) is time-independent, representing the steady state velocity (corresponding to the vorticity given in (9)) which is bounded everywhere except the origin for all times.

The nature of the singular perturbation for large time follows from (13). When the asymptotic properties of the modified Bessel function are utilized as we proceed to take the further limit $\tau \rho \rightarrow 0$, it is seen that

$$
\psi_{\text {oy }} \simeq-\frac{U}{4 \pi} \ln (\tau \rho)=-\frac{U}{4 \pi}(\ln \rho+\ln \tau) \text { for } \tau \rho \ll 1 ;
$$


it is seen that $\tau \rho \ll 1$ necessitates $|\ln \rho| \gg \ln \tau$. For this situation $U r / \nu$ represents the Reynolds number-it is variable because there is no natural length scale. The connection with the Stokes flow (4), from which we have

$$
\psi_{s y} \simeq-\frac{U}{4 \pi} \ln \rho \text { when } r \rightarrow \infty, t \rightarrow \infty, \text { with } \rho \rightarrow 0
$$

is clear.

Therefore, when we consider the solution for large times, the matching effectively requires that we take the limit $\rho \rightarrow 0$ in the Stokes equation solution, and the limit $\tau \rho \rightarrow 0$ in the Oseen equation solution.

A totally different approach towards the paradox is to see the two-dimensional Stokes flow as the limit of a three-dimensional Stokes flow when lengths in the $z$ direction tend to infinity. Some time ago, Shi [5] considered the steady low Reynolds number flow past an ellipsoid of revolution with large aspect ratio where the axis of revolution is set perpendicular to the uniform stream. He completed the singular perturbation analysis in the spirit of Kaplun and Lagerstrom [2], and Proudman and Pearson [3], showing how the Stokes solution was represented by the inner Oseen solution. His definition of the Reynolds number was based on the minor axis, and the results were still valid even as the major axis became large; the two-dimensional results for a circular cylinder could be retrieved in this manner.

Here we find first the unsteady Stokes flow due to a point stokeslet, and then through summation calculate the effect of a finite line distribution; the two-dimensional result follows on letting the length grow without bound. A similar tactic was recently used (Smith [6]) to gain an understanding of the Jeffery paradox. To begin, the point source of momentum is in the $x$-direction, positioned at the origin, and so we solve the equations

$$
\begin{aligned}
u_{x}+v_{y}+w_{z} & =0, \\
u_{t} & =-\rho^{-1} p_{x}+\nu \nabla^{2} u+\nu U \delta(x) \delta(y) \delta(z) H(t), \\
v_{t} & =-\rho^{-1} p_{y}+\nu \nabla^{2} v, \\
w_{t} & =-\rho^{-1} p_{z}+\nu \nabla^{2} w,
\end{aligned}
$$

where $(u, v, w)$ are the velocities in the $(x, y, z)$-directions. The solutions of these equations will be perturbations which are added to a uniform stream to approximate the flow past a small body. We define Laplace and Fourier transforms by

$$
\tilde{u}(\alpha, \beta, \gamma, s)=\int_{0}^{\infty} \cos \gamma z d z \int_{0}^{\infty} \cos \beta y d y \int_{0}^{\infty} \cos \alpha x d x \int_{0}^{\infty} e^{-s t} u(x, y, z, t) d t,
$$

with equivalent forms for $v, w$, and $p$, taking into account the different symmetries. We find from (15) that the solution of the transformed equations can be written as

$$
\tilde{u}_{p}=\frac{U\left(\beta^{2}+\gamma^{2}\right)}{s \Delta\left(\Delta+\frac{s}{v}\right)}, \quad \tilde{v}_{p}=\frac{U \alpha \beta}{s \Delta\left(\Delta+\frac{s}{v}\right)}, \quad \tilde{w}_{p}=\frac{U \alpha \gamma}{s \Delta\left(\Delta+\frac{s}{v}\right)},
$$


for $\Delta=\alpha^{2}+\beta^{2}+\gamma^{2}$. The inverse transforms can be evaluated from standard tables (c.f. Erdelyi [4]) for

$$
\begin{aligned}
u_{p}= & -\frac{2 \nu U}{\pi}\left(\frac{1}{R^{3}}-\frac{3 x^{2}}{R^{5}}\right)\left[t-\operatorname{terfc}\left\{\frac{R}{2(\nu t)^{1 / 2}}\right\}-\left(\frac{t}{\pi \nu}\right)^{1 / 2} \operatorname{Re}^{-R^{2} / 4 \nu t}\right] \\
& +\frac{U}{\pi}\left(\frac{1}{R}+\frac{x^{2}}{R^{3}}\right) \operatorname{erfc}\left\{\frac{R}{2(\nu t)^{1 / 2}}\right\}, \\
v_{p}= & \frac{6 \nu U x y}{\pi R^{5}}\left[t-\operatorname{terfc}\left\{\frac{R}{2(\nu t)^{1 / 2}}\right\}-\left(\frac{t}{\pi \nu}\right)^{1 / 2} \operatorname{Re}^{-R^{2} / 4 \nu t}\right] \\
& +\frac{U x y}{\pi R^{3}} \operatorname{erfc}\left\{\frac{R}{2(\nu t)^{1 / 2}}\right\},
\end{aligned}
$$

and

$$
\begin{aligned}
w_{p}= & \frac{6 \nu U x z}{\pi R^{5}}\left[t-\operatorname{terfc}\left\{\frac{R}{2(\nu t)^{1 / 2}}\right\}-\left(\frac{t}{\pi \nu}\right)^{1 / 2} \operatorname{Re}^{-R^{2} / 4 \nu t}\right] \\
& +\frac{U x z}{\pi R^{3}} \operatorname{erfc}\left\{\frac{R}{2(\nu t)^{1 / 2}}\right\} ;
\end{aligned}
$$

$R^{2}=x^{2}+y^{2}+z^{2}$. The steady state expressions which follow when $t \rightarrow \infty$ are

$$
u_{p \infty}=\frac{U}{\pi}\left(\frac{1}{R}-\frac{x^{2}}{R^{3}}\right), \quad v_{p \infty}=\frac{U x y}{\pi R^{3}}, \quad w_{p \infty}=\frac{U x z}{\pi R^{3}},
$$

as expected.

The next step is to consider the flow generated by a finite line distribution of stokeslets along the $z$-axis for $|z| \leq l$. There are two ways to proceed-either by re-solving Eqs. (15) when the delta function $\delta(z)$ is replaced by $H(z+l)-$ $H(z-l)$, where $H$ is the Heaviside step function, or, alternatively, by positioning a point stokeslet at $z=\xi$, and then integrating the slightly adjusted solutions already obtained with respect to $\xi$ for $|\xi| \leq l$. We follow the second procedure here, writing the velocities as $u_{l}, v_{l}, w_{l}$, where, for example,

$$
\begin{aligned}
u_{l}= & -\frac{2 \nu U}{\pi} \int_{-l}^{l}\left(\frac{1}{S^{3}}-\frac{3 x^{2}}{S^{5}}\right)\left[t-\operatorname{terfc}\left\{\frac{S}{2(\nu t)^{1 / 2}}\right\}-\left(\frac{t}{\pi \nu}\right)^{1 / 2} S e^{-s^{2} / 4 \nu t}\right] d \xi \\
& +\frac{U}{\pi} \int_{-l}^{l}\left(\frac{1}{S}+\frac{x^{2}}{S^{3}}\right) \operatorname{erfc}\left\{\frac{S}{2(\nu t)^{1 / 2}}\right\} d \xi
\end{aligned}
$$

$S^{2}=x^{2}+y^{2}+(z-\xi)^{2}$. Neither this integral, nor the corresponding ones for $v_{l}$ and $w_{l}$ can be simplified sufficiently to give general information which is of value, though different approximations are possible to give the required understandings.

First, we note that when $l \rightarrow \infty$ the integrals can be evaluated to reproduce the two-dimensional results already given in (4). The next observation is that when 
the limit of (20) is taken (for finite $l$ ) as $t \rightarrow \infty$, then bounded velocities for all $x, y, z, t$ are evident; specifically,

$$
\begin{aligned}
u_{l \infty}=\frac{U}{\pi} & {\left[\ln \frac{\left\{r^{2}+(z+l)^{2}\right\}^{1 / 2}+(z+l)}{\left\{r^{2}+(z-l)^{2}\right\}^{1 / 2}+(z-l)}\right.} \\
& \left.+\frac{x^{2}}{r^{2}}\left(\frac{z+l}{\left\{r^{2}+(z+l)^{2}\right\}^{1 / 2}}-\frac{z-l}{\left\{r^{2}+(z-l)^{2}\right\}^{1 / 2}}\right)\right], \\
v_{l \infty}= & \frac{U x y}{\pi r^{2}}\left[\frac{z+l}{\left\{r^{2}+(z+l)^{2}\right\}^{1 / 2}}-\frac{z-l}{\left\{r^{2}+(z-l)^{2}\right\}^{1 / 2}}\right], \\
w_{l \infty}= & -\frac{U x}{\pi r^{2}}\left[\frac{1}{\left\{r^{2}+(z+l)^{2}\right\}^{1 / 2}}-\frac{1}{\left\{r^{2}+(z-l)^{2}\right\}^{1 / 2}}\right] .
\end{aligned}
$$

In the particular case that the limit as $l \rightarrow \infty$ is taken for these steady state velocities, we have

$$
\begin{gathered}
\lim _{l \rightarrow \infty}\left\{u_{l \infty}-\frac{2 U}{\pi} \ln (2 l)\right\}=\frac{2 U}{\pi}\left\{\frac{x^{2}}{r^{2}}-\ln r\right\}, \\
\lim _{l \rightarrow \infty} v_{l \infty}=\frac{2 U x y}{\pi r^{2}}, \quad \lim _{l \rightarrow \infty} w_{l \infty}=0,
\end{gathered}
$$

with an error which is $O\left(l^{-2}\right)$; there is a singular behaviour as the length of the line distribution $l$ grows without bound.

In fact, if we take the limits as $t \rightarrow \infty$ and then $l \rightarrow \infty$ in (20) there is the $\ln l$ singularity noted above, whereas if we reverse the order and take the limits as $l \rightarrow \infty$, and then $t \rightarrow \infty$ in (2) there is the $\ln t$ singularity observed from the integral

$$
I=\int_{-l}^{l} \frac{1}{S} \operatorname{erfc}\left\{\frac{S}{2(\nu t)^{1 / 2}}\right\} d \xi=\int_{z-l}^{z+l} \frac{1}{\left(r^{2}+\beta^{2}\right)^{1 / 2}} \operatorname{erfc}\left\{\frac{\left(r^{2}+\beta^{2}\right)^{1 / 2}}{2(\nu t)^{1 / 2}}\right\} d \beta,
$$

and we now consider this integral when both $l$ and $t$ are large, but $r, z=O(1)$. Straightforward integration by parts shows first, when $l \gg 1$ but $l /(\nu t)^{1 / 2} \ll 1$, that

$$
I \simeq \ln l+(\text { finite terms })-2 l(\pi \nu t)^{-1 / 2}
$$

alternatively, if $l /(\nu t)^{1 / 2}=O(1)$, or larger, then the $\ln t$ behaviour is also present. Therefore it is clear that however large the value of (noninfinite) $l$, when the time $t$ itself is infinite there is definitely a steady state solution-even though the $\ln l$ term still remains. In the context of a Stokes flow the behaviour found earlier in (4) is the direct consequence of taking the limit as $l \rightarrow \infty$ first, followed by $t \rightarrow \infty$; when the order is commuted the unbounded velocity is no longer evident.

Embedding the first part of the paper in the historical context of Stokes' classical work follows the suggestion of a referee, and the author wishes to acknowledge this assistance. A grant in aid of research was received from the Natural Sciences and Engineering Research Council of Canada during the time this work was completed. 


\section{REFERENCES}

[1] G. G. Stokes, On the effect of the internal friction of fluids on the motion of pendulums, Trans. Camb. Phil. Soc. 9, 9-106 (1851)

[2] S. Kaplun and P. A. Lagerstrom, Asymptotic expansions of Navier-Stokes solutions for small Reynolds numbers, J. Math. Mech. 6, 585-593 (1957)

[3] I. Proudman and J. R. A. Pearson, Expansions at small Reynolds numbers for the flow past a sphere and a circular cylinder, J. Fluid Mech. 2, 237-262 (1957)

[4] A. Erdelyi, et al., Tables of Integral Transforms, Vol. 1, McGraw-Hill, New York, 1954

[5] Y. Y. Shi, Low Reynolds number flow past an ellipsoid of revolution of large aspect ratio, J. Fluid Mech. 23, 657-671 (1965)

[6] S. H. Smith, The Jeffery paradox as the limit of a three dimensional Stokes flow, Phys. Fluids A. 2, 661-665 (1990) 